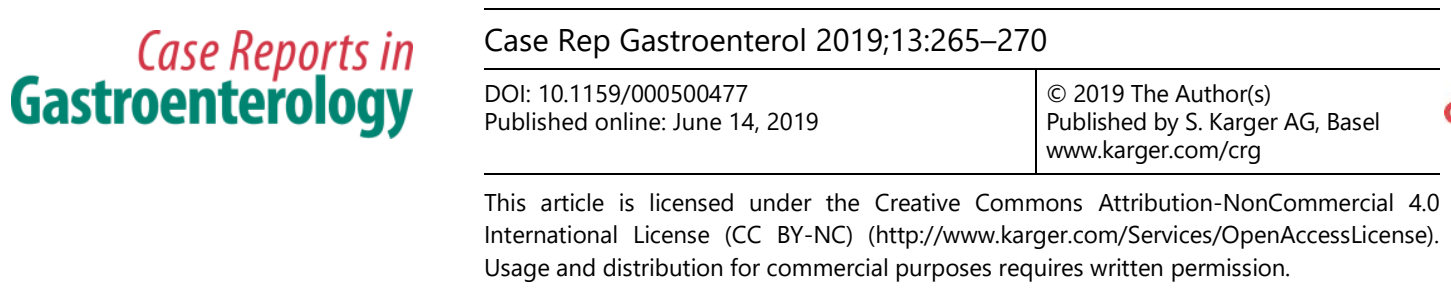

\title{
Type 1 Autoimmune Pancreatitis with Imaging Appearance Similar to That of Malignant Cystic Tumor
}

\author{
Takeshi Ezaki $^{a} \quad$ Atsuhiro Masuda ${ }^{a}$ Hideyuki Shiomi ${ }^{a}$ \\ Takashi Nakagawa $^{a} \quad K^{2}$ itaro Sofue ${ }^{b}$ Hirochika Toyama $^{c}$ Yoh Zen $^{d}$ \\ Yuzo Kodama ${ }^{\mathrm{a}}$ \\ aDivision of Gastroenterology, Department of Internal Medicine, Kobe University Graduate \\ School of Medicine, Kobe, Japan; 'b Department of Radiology, Kobe University Graduate \\ School of Medicine, Kobe, Japan; 'Division of Hepato-Biliary-Pancreatic Surgery, \\ Department of Surgery, Kobe University Graduate School of Medicine, Kobe, Japan; \\ dDivision of Diagnostic Pathology, Kobe University Graduate School of Medicine, \\ Kobe, Japan
}

\section{Keywords}

Autoimmune pancreatitis - Intraductal papillary mucinous carcinoma - Pancreas · Pancreatic cyst

\begin{abstract}
A 79-year-old man was admitted with asymptomatic elevation of liver enzymes and tumor markers. Abdominal contrast-enhanced computed tomography demonstrated swelling of the pancreatic head, and additional blood test showed raised IgG4 levels. Histological examination by endoscopic ultrasonography (EUS)-guided fine needle aspiration for pancreatic head mass revealed storiform fibrosis and IgG4-positive plasma cell infiltration. We diagnosed this case as type 1 autoimmune pancreatitis (AIP). In addition, there was a cystic lesion in the pancreatic body apart from the pancreatic head mass. A mural nodule in the multilocular cyst was detected by EUS, and there was positive uptake of fluorodeoxyglucose in positron emission tomography/magnetic resonance imaging. The preoperative diagnosis of this cystic lesion was intraductal papillary mucinous carcinoma, and distal pancreatomy was performed. Histopathological findings showed various sizes of retention cysts caused by IgG4-positive plasma cell
\end{abstract}


infiltration around the pancreatic branch ducts. The mural nodule was a fibrotic mass with diffuse infiltration of IgG4-positive cells. This cystic lesion mimicking malignant cystic neoplasm occurred in relation to AIP. This case provided important information helping to understand the mechanism of formation of mural nodules in multilocular cysts in patients with type 1 AIP.

\section{Introduction}

Autoimmune pancreatitis (AIP) is chronic inflammatory pancreatitis associated with autoimmune manifestation. It is known that AIP rarely causes cystic lesions in the pancreas. Most of these cystic lesions have been reported to be pseudocysts or retention cysts caused by pancreatic duct stenosis with severe lymphoplasmacytic infiltration in the periductal area $[1,2]$. However, it has also been reported that neoplastic cystic lesions sometimes coexist with AIP, which are intraductal papillary mucinous neoplasm or mucinous neoplasm [3, 4]. They often showed images similar to those of nonneoplastic cysts. Therefore, in most cases, it is difficult to distinguish neoplastic from nonneoplastic cysts. Herein, we describe an AIP case with mimicking neoplastic cyst and compare pathological and preoperative images.

\section{Case Report}

A 79-year-old male with a previous history of cholecystectomy and endoscopic choledocholithotomy was admitted with asymptomatic elevation of liver enzymes and tumor markers. Initial laboratory values showed total bilirubin at $1.1 \mathrm{mg} / \mathrm{dL}$ (normal $0.4-1.5$ ), alkaline phosphatase at 1,544 U/L (normal 106-322), gamma-glutamyltranspeptidase at 1,031 U/L (normal 13-64), aspartate aminotransferase at $82 \mathrm{U} / \mathrm{L}$ (normal 13-30), alanine aminotransferase at $107 \mathrm{U} / \mathrm{L}$ (normal 10-42), carcinoembryonic antigen at $5.8 \mathrm{ng} / \mathrm{mL}$ (normal <5), carbohydrate-associated antigen $19-9$ at $142 \mathrm{U} / \mathrm{mL}$ (normal <37), s-pancreas-1 antigen at $302 \mathrm{U} / \mathrm{mL}$ (normal <30), and pancreatic cancer-associated antigen at $61 \mathrm{U} / \mathrm{mL}$ (normal < 150). Abdominal contrast-enhanced computed tomography demonstrated swelling of the pancreatic head and mild dilation of the intrahepatic and extrahepatic bile duct (Fig. 1a, b), and an additional blood test showed high IgG4 levels ( $774 \mathrm{mg} / \mathrm{dL}$; normal 5-117). Endoscopic retrograde cholangiopancreatography revealed segmental narrowing of the main pancreatic duct in the pancreatic head without distal main pancreatic duct dilation and stenosis of the lower bile duct. Intraductal ultrasonography showed bile duct wall thickening with bile duct stricture. Bile duct biopsy did not show neoplastic change. Furthermore, histological examination by endoscopic ultrasonography (EUS)-guided fine needle biopsy for the pancreatic head mass revealed storiform fibrosis and IgG4-positive plasma cell infiltration. We diagnosed this case as type 1 AIP and IgG4-related sclerosing cholangitis.

In addition, there was a multilocular cystic lesion (maximum diameter $15 \mathrm{~mm}$ ) in the pancreatic body apart from the pancreatic head swelling (Fig. 1a). Endoscopic retrograde cholangiopancreatography showed that the cyst communicated with the main pancreatic duct. Additionally, a mural nodule (maximum diameter $5 \mathrm{~mm}$ ) was detected by EUS in the cyst (Fig. 1c, left panel), and that was enhanced by contrast-enhanced EUS (Fig. 1c, right panel). That lesion also showed positive uptake of fluorodeoxyglucose (FDG) in positron emission tomography/magnetic resonance imaging with a maximum standardized uptake value of 4.16 
(Fig. 1b). Considering these results, our preoperative diagnosis of this cystic lesion was intraductal papillary mucinous carcinoma, and distal pancreatomy was performed.

Histopathological findings showed various sizes of retention cysts, which were lined by nonneoplastic pancreatic epithelium (Fig. 2a). These cysts were the dilated pancreatic branch ducts with diffuse infiltration of IgG4-positive plasma cells. The mural nodule was a fibrotic mass with severe infiltration of IgG4-positive cells (>100 IgG4-positive plasma cells per highpower field, IgG4+/IgG+ plasma cell ratio $65.6 \%$; Fig. 2b). Neoplastic change was not seen in the main and branch pancreatic ducts. The adjacent pancreatic parenchyma appeared to be atrophic with only mild inflammation, but foci of storiform fibrosis and obliterative phlebitis were observed, leading to the diagnosis of partly regressed AIP with residual periductal inflammation.

\section{Discussion and Conclusion}

AIP is a rare chronic inflammatory pancreatitis associated with autoimmune manifestation, which was first described by Sarles et al. [5] as hypergammaglobulinemia-associated chronic pancreatitis in 1961 and first named by Yoshida et al. [6] as AIP in 1995. Furthermore, Hamano et al. [7] firstly reported that serum IgG4 levels are significantly and specifically high in AIP patients. In 2011, the International Consensus Diagnostic Criteria proposed two types of AIP. Type 1 AIP is associated with histological feature called lymphoplasmacytic sclerosing pancreatitis with elevated serum IgG4, type 2 is characterized by idiopathic duct-centric pancreatitis with granulocytic epithelial lesion and serum IgG4 negativity [8]. In this case, pathological findings of lymphoplasmacytic sclerosing pancreatitis and serum IgG4 elevation were found, and that led to the diagnosed of type 1 AIP.

There have been only few reports of pancreatic cystic lesions associated with AIP. Most of these cystic lesions were reported to be located at the pancreatic body to tail, and they were pseudocysts or retention cysts caused by pancreatic duct stenosis with severe lymphoplasmacytic infiltration in the periductal area [1, 2]. It was also reported that neoplastic cystic lesions sometimes coexist with AIP, which are intraductal papillary mucinous neoplasm or mucinous neoplasm $[3,4]$. They often showed images similar to those of nonneoplastic cysts. Therefore, in most cases, it is difficult to distinguish neoplastic from nonneoplastic cysts. In our case, it was hard to diagnose this lesion as nonneoplastic cyst even in retrospect.

In the present case, pathological examination revealed that the cystic lesion of the pancreatic body was a retention cyst lined by normal pancreatic epithelium without neoplastic changes. Although microscopic findings consistent with type 1 AIP (e.g., IgG4-positive cell infiltration, storiform fibrosis, and obliterative phlebitis) were observed, the degree of inflammation was markedly lower than that expected in untreated AIP. As shown in Figure 2, EUS findings correlated well with the microscopic appearance. Intense lymphoplasmacytic infiltration was mainly noted in the subepithelial layer of the dilated duct, the histological finding likely corresponding to the FDG-positive mural nodule. Although type 1 AIP is typically a lobule-centric inflammatory process, parenchymal inflammation had already regressed at the initial presentation in the present case, which is probably the reason why imaging features were atypical for type 1 AIP.

It has been reported that AIP shows prompt response to corticosteroid treatment, usually within 1-4 weeks $[9,10]$. In previous reports, most cases with cystic lesions associated with AIP also showed rapid response to corticosteroid treatment [1]. Especially, in case of cysts with a diameter $<55 \mathrm{~mm}, 100 \%$ of patients improved with corticosteroid treatment within 
4 weeks [2]. Corticosteroids may release the stasis of the pancreatic juice caused by pancreatic ductal stenosis due to the lymphoplasmacytic infiltration $[1,2]$. Cystic lesions associated with AIP could be treated by corticosteroid therapy rather than interventional treatment as a firstline treatment.

A correct preoperative diagnosis is important to avoid unnecessary surgery. In our case, the cystic lesion looked like intraductal papillary mucinous carcinoma because of the enhanced mural nodule in contrast-enhanced EUS with instinct FDG uptake in positron emission tomography/magnetic resonance imaging. For suspected malignant tumor, there is still hesitation to use corticosteroids for differential diagnosis. In differentiating focal-type AIP from pancreatic cancer, some reports showed that steroid trial of only 2 weeks was effective [11, 12]. Therefore, even in our case, short-term steroid trial might be a potential treatment for differential diagnosis. However, it remains inconclusive to use corticosteroids for cystic lesions with malignant features in patients with AIP.

\section{Statement of Ethics}

The patient gave his informed consent before publication.

\section{Disclosure Statement}

The authors have no conflict of interest.

\section{Funding Sources}

This study did not receive direct funding support.

\section{References}

1 Muraki T, Hamano H, Ochi Y, Arakura N, Takayama M, Komatsu K, et al. Corticosteroid-responsive pancreatic cyst found in autoimmune pancreatitis. J Gastroenterol. 2005 Jul;40(7):761-6.

2 Kubota K, Fujita Y, Sato T, Sekino Y, Hosono K, Kobayashi N, et al. Autoimmune pancreatitis associated with pancreatic cyst: how can we manage it? J Hepatobiliary Pancreat Sci. 2014 Dec;21(12):902-10.

3 Tabata T, Kamisawa T, Hara S, Kuruma S, Chiba K, Kuwata G, et al. Intraductal papillary mucinous neoplasm of the pancreas and IgG4-related disease: a coincidental association. Pancreatology. 2013 Jul-Aug;13(4): 379-83.

4 Matsubayashi H, Iwai T, Matsui T, Wada T, Kawata N, Ito H, et al. Pancreatic cystic lesions with atypical steroid response should be carefully managed in cases of autoimmune pancreatitis. J Gastroenterol Hepatol. 2016 Jan;31(1):270-6.

5 Sarles H, Sarles JC, Muratore R, Guien C. Chronic inflammatory sclerosis of the pancreas - an autonomous pancreatic disease? Am J Dig Dis. 1961 Jul;6(7):688-98.

6 Yoshida K, Toki F, Takeuchi T, Watanabe S, Shiratori K, Hayashi N. Chronic pancreatitis caused by an autoimmune abnormality. Proposal of the concept of autoimmune pancreatitis. Dig Dis Sci. 1995 Jul;40(7): 1561-8.

7 Hamano H, Kawa S, Horiuchi A, Unno H, Furuya N, Akamatsu T, et al. High serum IgG4 concentrations in patients with sclerosing pancreatitis. N Engl J Med. 2001 Mar;344(10):732-8.

8 Shimosegawa T, Chari ST, Frulloni L, Kamisawa T, Kawa S, Mino-Kenudson M, et al.; International Association of Pancreatology. International consensus diagnostic criteria for autoimmune pancreatitis: guidelines of the International Association of Pancreatology. Pancreas. 2011 Apr;40(3):352-8.

9 Kamisawa T, Shimosegawa T, Okazaki K, Nishino T, Watanabe H, Kanno A, et al. Standard steroid treatment for autoimmune pancreatitis. Gut. 2009 Nov;58(11):1504-7. 


\begin{tabular}{l|l}
\hline Case Rep Gastroenterol 2019;13:265-270 \\
\hline DOI: 10.1159/000500477 & $\begin{array}{l}\text { @ 2019 The Author(s). Published by S. Karger AG, Basel } \\
\text { www.karger.com/crg }\end{array}$ \\
\hline
\end{tabular}

Ezaki et al.: Type 1 Autoimmune Pancreatitis with Imaging Appearance Similar to That of Malignant Cystic Tumor

10 Matsubayashi H, Yoneyama M, Nanri K, Sugimoto S, Shinjo K, Kakushima N, et al. Determination of steroid response by abdominal ultrasound in cases with autoimmune pancreatitis. Dig Liver Dis. 2013 Dec;45(12): 1034-40.

11 Moon SH, Kim MH, Park DH, Hwang CY, Park SJ, Lee SS, et al. Is a 2-week steroid trial after initial negative investigation for malignancy useful in differentiating autoimmune pancreatitis from pancreatic cancer? A prospective outcome study. Gut. 2008 Dec;57(12):1704-12.

12 Tomiyama T, Uchida K, Matsushita M, Ikeura T, Fukui T, Takaoka M, et al. Comparison of steroid pulse therapy and conventional oral steroid therapy as initial treatment for autoimmune pancreatitis. Gastroenterol. 2011 May;46(5):696-704.
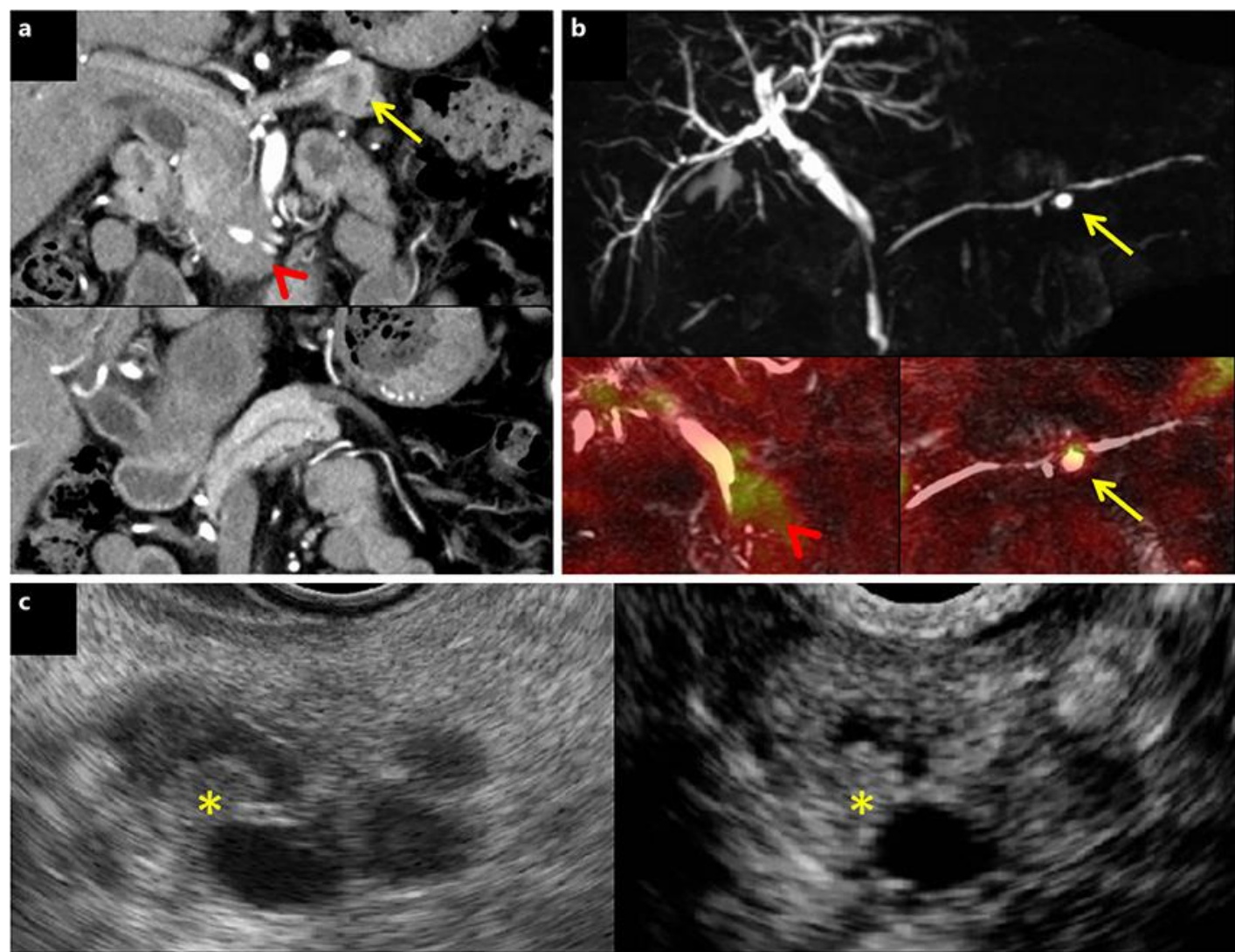

Fig. 1. Preoperative imaging studies. a Contrast-enhanced computed tomography showed swelling of pancreatic head (upper panel, red arrowhead) and the cystic lesion in pancreatic body (upper panel, yellow arrow). Except for these parts, the pancreas was intact (lower panel). b Magnetic resonance cholangiopancreatography demonstrated strictures of the main pancreatic duct in the pancreatic head and pancreas tail which was accompanied by a cystic lesion. Positron emission tomography/magnetic resonance imaging showed marked fluorodeoxyglucose uptake both in the pancreatic head (maximum standardized uptake value 7.12) (lower left panel, red arrowhead) and the cystic lesion of the pancreatic body (maximum standardized uptake value 4.16) (upper and lower right panel, yellow arrow). c EUS detected the mural nodule (asterisk) in the cystic lesion (left panel), which was enhanced by contrast-enhanced EUS (right panel). EUS, endoscopic ultrasonography. 


\section{Case Reports in Gastroenterology}
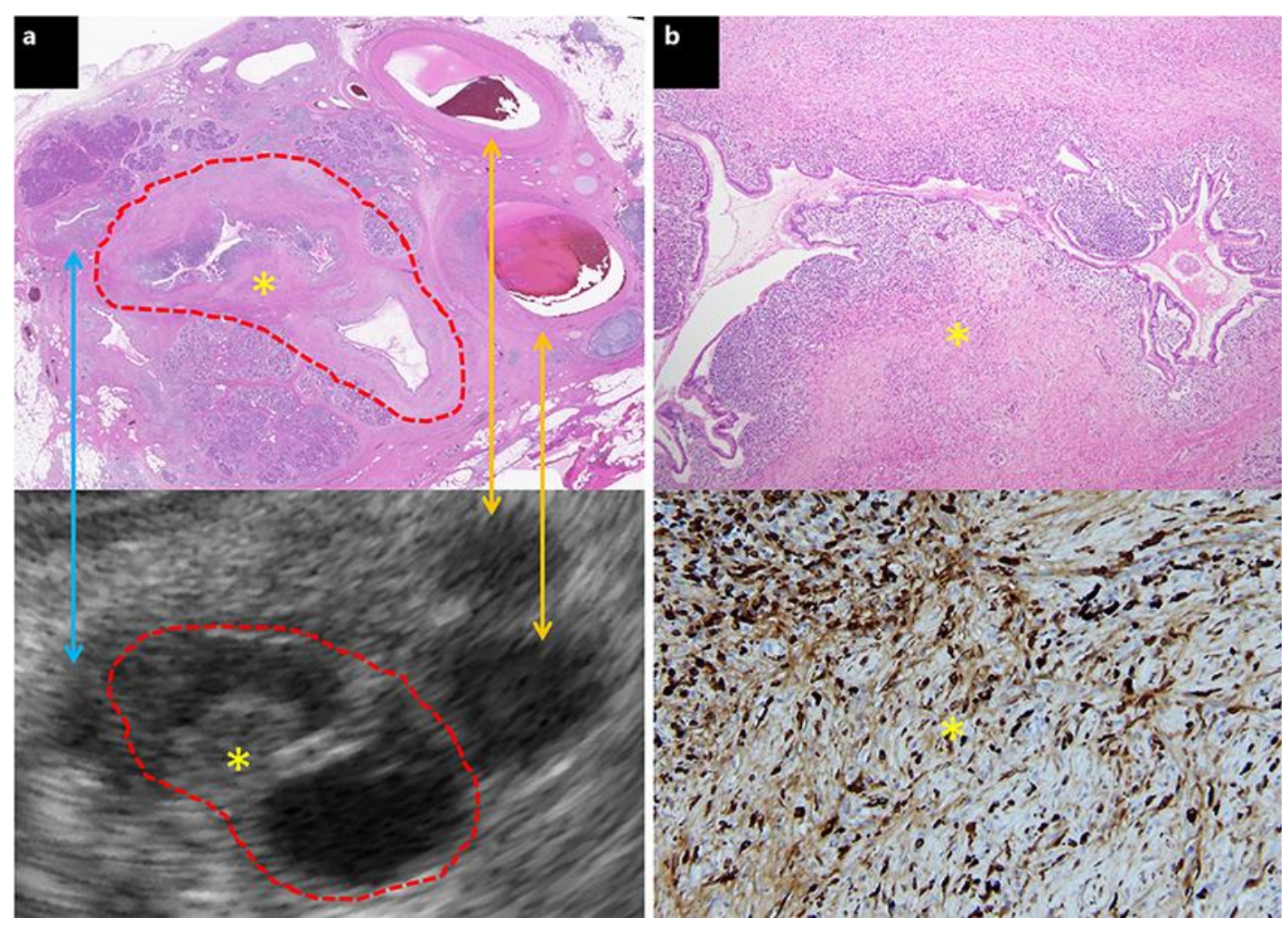

Fig. 2. Comparison of pathological image and EUS image. a Loupe view of the resected specimen showed that the cystic lesion was a retention cysts lined by normal epithelium (upper panel, H\&E stain). The pathological image was finely in accordance with the EUS image (lower panel). The area circled by red dots is a retention cyst with inflammatory cell infiltration. The asterisk is a mural nodule detected by EUS. The blue arrow shows the main pancreatic duct and the yellow arrows show the splenic artery and vein. $\mathbf{b}$ The mural nodule (asterisk) was a fibrotic mass with IgG4-positive lymphoplasmacytic infiltration (upper panel: H\&E stain, 40× magnification; lower panel: IgG4 stain, 400× magnification). EUS, endoscopic ultrasonography. 